\title{
Predicting human papillomavirus vaccine uptake in young adult women: Comparing the Health Belief Model and Theory of Planned Behavior
}

\author{
Mary A. Gerend and Janet E. Shepherd \\ Florida State University College of Medicine
}

\section{Abstract}

Background-Although theories of health behavior have guided thousands of studies, relatively few studies have compared these theories against one another.

Purpose-The purpose of the current study was to compare two classic theories of health behavior - the Health Belief Model (HBM) and the Theory of Planned Behavior (TPB) - in their prediction of human papillomavirus (HPV) vaccination.

Methods-After watching a gain-framed, loss-framed, or control video, women ( $N=739)$ ages 18-26 completed a survey assessing HBM and TPB constructs. HPV vaccine uptake was assessed ten months later.

Results-Although the message framing intervention had no effect on vaccine uptake, support was observed for both the TPB and HBM. Nevertheless, the TPB consistently outperformed the HBM. Key predictors of uptake included subjective norms, self-efficacy, and vaccine cost.

Conclusions-Despite the observed advantage of the TPB, findings revealed considerable overlap between the two theories and highlighted the importance of proximal versus distal predictors of health behavior.

\section{Keywords}

Theory testing; Health behavior theory; Cervical cancer prevention; Vaccination

People's decisions to engage in health protective behaviors are influenced by a variety of psychological factors (e.g., attitudes, beliefs, intentions), many of which are represented in major theories of health behavior [1-5]. Such theories are useful not only because they help understand and predict health behavior, but also because they guide interventions by identifying potentially modifiable antecedents of health behavior [6-7]. The purpose of the current study was to compare the predictive ability of two of the most widely used theories of health behavior - the Health Belief Model (HBM) $[2,8]$ and the Theory of Planned Behavior (TPB) [1]. Although theories of health behavior have guided thousands of studies, surprisingly little is known about the relative efficacy of these theories in predicting health behavior. Indeed, to truly advance the literature, scholars have called for research that not only rigorously tests health behavior theories, but also directly compares them against one

Address correspondence and reprint requests to: Mary A. Gerend, Department of Medical Humanities and Social Sciences, College of Medicine, Florida State University, 1115 West Call Street, Tallahassee, Florida 32306-4300. Phone: 850-645-1542; Fax:

850-645-1773; mary.gerend@med.fsu.edu.

Conflict of Interest Statement: The authors have no conflict of interest to disclose. 
another [9-10]. The current study sought to address this gap by comparing the ability of two classic theories in predicting human papillomavirus (HPV) vaccination.

The HBM was originally developed in the 1950s to predict whether individuals engaged in programs to prevent and detect disease [2,5]. The HBM consists of five constructs proposed to influence the likelihood an individual will engage in a given health behavior to avoid an undesirable health outcome. They include: perceived susceptibility (perceptions of the likelihood that one will experience the outcome), perceived severity (perceptions of the seriousness of the consequences associated with the outcome), perceived benefits (potential advantages of engaging in the health behavior, including the behavior's perceived efficacy in preventing the undesired outcome), and perceived barriers (perceived obstacles to engaging in the health behavior). Cues to action, the fifth and least studied construct in the HBM, are factors that signal or remind an individual to engage in the health behavior and range from experiencing symptoms to receiving persuasive appeals from the media, family and friends, or a health care provider.

The TPB is an extension of the Theory of Reasoned Action [4], and has been used to predict behaviors across a wide variety of domains including health [11]. The Theory of Reasoned Action proposes that attitudes and subjective norms lead to the formation of behavioral intentions which, in turn, serve as proximal causes of behavior. Attitudes are defined as the product of beliefs about whether performing the behavior leads to positive and negative outcomes and evaluations of those outcomes. Subjective norms reflect people's beliefs about whether important others think they should perform the behavior and their motivation to comply with those recommendations. Ajzen [1] later added the construct of perceived behavioral control to the Theory of Reasoned Action after which it became known as the TPB. Perceived behavioral control is a multidimensional construct that reflects perceived control over the behavior, perceived difficulty of engaging in the behavior, and perceived self-efficacy to perform the behavior. Both the Theory of Reasoned Action and TPB hold that attitudes and subjective norms influence behavior indirectly through intentions, such that intentions are the direct precursor of behavior. Perceived behavioral control is hypothesized to affect behavior both directly and indirectly through its effects on intentions.

When comparing the HBM and TPB, it is useful to consider their similarities and differences. The HBM and TPB share several features in common. Both theories adopt an individual-level approach to predicting health behavior and both are based on an expectancy-value framework [12]. Further, both theories assume that health decisionmaking is a largely deliberative and rational process $[6-7,12]$. There are also notable similarities in the specific constructs associated with the two theories (e.g., see Weinstein [10] for a detailed review.) The self-efficacy component of perceived behavioral control in the TPB, for example, overlaps with perceived barriers from the HBM, in that items used to assess self-efficacy typically reflect people's confidence in their ability to perform the health behavior in the presence of various barriers (e.g., How confident are you that you will be able to exercise tomorrow even if it's raining?)[10]. Similarly, although cues to action are not formally represented in the TPB, they may be indirectly reflected in people's attitudes and subjective norms about a given health behavior, as attitudes and norms could be shaped by discussions with friends and health care providers or recent exposures to media campaigns [6].

The HBM and TPB are also characterized by important differences. The primary difference between the two theories is that, unlike the HBM, the TPB contains a proximate predictor of behavior: behavioral intentions. The TPB posits that behavioral intentions are the immediate antecedent of behavior and thus the best predictor of behavior. Although intentions were not included in the original conceptualization of the HBM, some have argued that it may be 
appropriate to consider health beliefs (e.g., perceived susceptibility, barriers) as antecedents of intentions rather than direct predictors of behavior [6]; in this view, HBM constructs may operate via intentions, as TPB constructs are presumed to do. Another important difference between the two theories is that, unlike the TPB, the HBM traditionally did not include a component that directly captured perceptions of control or self-efficacy. However, due to its success in predicting behavior, self-efficacy was added to later renditions of the HBM [5, 13].

Despite clear similarities and differences between these two theories, relatively few studies have compared them against one another [9-10]. Indeed, this lack of comparative theory testing applies to the health behavior literature more generally. Noar and Zimmerman [9] identified only 19 studies that formally compared two or more theories of health behavior. We updated this search through February of 2011 and found only a few more [14-16]. As noted by others $[12,17]$, most studies comparing health behavior theories are limited in that they tend to rely on cross-sectional data and/or examine intentions (rather than behavior) as the primary outcome variable. The current work advances the literature in part by using a longitudinal design and by including a behavioral outcome.

Previous studies assessing the relative efficacy of different health behavior theories have tended to do so by comparing the percentage of variance explained in behavior as a way of determining which theory is more effective [17]. We adopted a similar approach in the current work, although we sought to take it a step further. Because most individual-level theories of health behavior include overlapping constructs [10], there is value to combining theories to identify which specific constructs are able to account for unique variance in behavior. This strategy can also assist in evaluating the extent to which constructs from the two theories account for the same versus distinct variance in health behavior $[9,17]$. Based on this reasoning, we estimated a combined model that simultaneously included constructs from the HBM and TPB to examine the extent of overlap across the two theories and to identify independent predictors.

The health behavior of interest for the current investigation was HPV vaccination, a primary prevention strategy for infections known to cause cervical cancer. Approved by the U.S. Food and Drug Administration (FDA) in June of 2006, the quadrivalent HPV vaccine (Gardasil, Merck and Co. Inc) provides protection against four types of HPV transmitted during sexual activity: two oncogenic types (HPVs 16 and 18) responsible for approximately $70 \%$ of all cases of cervical cancer, and two non-oncogenic types (HPVs 6 and 11) responsible for $90 \%$ of all cases of genital warts [18]. A bivalent HPV vaccine (Cervarix, GlaxoSmithKline) targeting HPV types 16 and 18 received FDA approval in October of 2009. Both vaccines have demonstrated high efficacy in preventing high-grade cervical intraepithelial neoplasia, a known precursor of cervical cancer, and have favorable safety and tolerance profiles [19-21]. HPV vaccination is recommended for females ages 11 and 12 , with "catch-up" vaccination recommended for females up to 26 years of age [19]. Both vaccines consist of three doses administered (ideally) over a six-month period.

Research on the predictors of HPV vaccine uptake is beginning to accumulate; however, the majority of studies have examined predictors of HPV vaccine acceptability (i.e., people's intentions or willingness to vaccinate themselves or their children [22-23]) rather than HPV vaccination behavior itself. A review by Brewer and Fazekas [22] found significant relationships between HPV vaccine acceptability and most HBM constructs (i.e., perceived susceptibility, perceived benefits and effectiveness, perceived barriers, and physician recommendation as a cue to action). Of the few studies examining predictors of behavior (i.e., HPV vaccine uptake), subjective norms, perceived barriers, perceived susceptibility to 
HPV infection, and physician recommendation have been associated with receipt of one or more HPV injections [24-29].

Data from the present study were drawn from a randomized clinical trial investigating the effects of framed health messages on vaccine uptake. Although the message framing intervention had no effect on HPV vaccine uptake, the study provided an opportunity to test the utility of the HBM versus TPB in predicting uptake among young adult women. Using structural equation modeling, separate models were estimated for the HBM and TPB, both with and without behavioral intentions as a proximal mediating variable. By estimating models with and without intentions, we were able to examine the extent to which more distal constructs predict behavior directly [30]. Additionally, to advance understanding of the often neglected cues to action construct, we tested the hypothesis that in addition to predicting behavior directly, cues to action might also affect intentions and behavior indirectly via people's health beliefs [6]. Having a doctor recommend the HPV vaccine could, for example, increase an individual's perception of susceptibility to HPV infection, which could subsequently increase intentions to receive the vaccine. Finally, to provide more insight into the extent of overlap in variance accounted for by HBM and TPB constructs and to identify independent predictors of vaccine uptake, we estimated a combined model in which uptake was predicted from HBM and TPB constructs concurrently.

\section{Method}

\section{Participants}

Young adult women $(N=739)$ attending a large southeastern university in the U.S. were recruited via flyers, online announcements on the university's course management system, and the Department of Psychology subject pool. Interested participants were directed to a webpage to take a screening survey. A total of 2,782 women completed the screening survey, of whom $58 \%(n=1,612)$ met eligibility criteria (female; aged 18-26 years; no prior receipt of any doses of the HPV vaccine; not currently pregnant). Most ineligible respondents had already been vaccinated $(n=888)$ or fell outside the $18-26$ age range $(n=$ 222). All eligible participants were invited to enroll in the intervention study and data collection continued until the desired sample size $(N=735)$ was reached. Sample size was determined by a power analysis in which the intervention was expected to have a small-tomedium effect (Cohen's $\mathrm{h}=.30$ to .40 ) on vaccine uptake.

Participants are described in detail elsewhere [31-32]. Mean age was 21 years $(S D=1.8)$. Sixteen percent were Hispanic or Latina. The majority of participants self-identified as White (68\%) or Black or African American (19\%). Most participants were in their junior or senior year of college $(61 \%)$. The majority of participants $(91 \%)$ had some form of health insurance, with over two thirds covered by their parents' health insurance. Nearly $80 \%$ had previously engaged in vaginal intercourse. At baseline, about half (49\%) of participants had received a recommendation from their physician or another health care provider for the HPV vaccine.

\section{Procedure}

Data were drawn from a randomized clinical trial, the purpose of which was to assess the relative efficacy of three educational videos on young adult women's uptake of HPV vaccine. At baseline, participants were randomly assigned to view a gain-framed $(n=250)$, loss-framed $(n=243)$, or control (no framed information; $n=246$ ) video about HPV vaccination. The gain-framed video emphasized the benefits of getting vaccinated ("By receiving the HPV vaccine you will decrease your chances of getting an unwanted HPV infection."), whereas the loss-framed video emphasized the costs of not getting vaccinated 
("By not receiving the HPV vaccine you will increase your chances of getting an unwanted HPV infection."). Other than message frame, content was essentially identical across the three conditions. Content was drawn from the Centers for Disease Control and Prevention's website and previous research [33-34]. Each video included detailed information about HPV infection (e.g., prevalence, risk factors, health consequences, methods of diagnosis) and the quadrivalent HPV vaccine (e.g., efficacy, safety profile, dosing schedule). The video also provided information on vaccine cost $(\$ 120-\$ 160$ per dose), coverage of the vaccine by health insurance plans and other programs (e.g., Vaccines for Children), and local distribution centers such as the student health center on campus, which is open year round. Before dismissal, participants received a pamphlet that contained all of the information presented in the video and a list of local clinics that offered the vaccine.

The baseline session took place in a laboratory on campus in groups of up to five participants. After providing informed consent participants completed a brief pre-test survey assessing sexual history and knowledge of HPV, viewed a video about HPV vaccination, and completed a post-test survey assessing HBM and TPB constructs and background characteristics. Baseline data collection took place from March through August of 2009. For completing the baseline session participants recruited from the general student body ( $n=$ 692) received $\$ 30$ and participants recruited through the Department of Psychology $(n=47)$ received course credit.

Ten months later (January through June 2010), participants received an email invitation with a secure link to the online follow-up survey. The primary outcome variable (HPV vaccine uptake) was assessed on the follow-up assessment. In all, 696 (94\%) participants completed follow-up, for which they received a $\$ 10$ electronic gift card. Participants who had not completed the survey after four additional reminder emails were contacted by a research assistant. All study materials and procedures were approved by the University Human Subjects Committee.

\section{Measures}

HBM and TPB constructs-HBM and TPB constructs were assessed with multiple items and were drawn from previous research whenever possible (see Table 1 for example items and the electronic supplementary material (ESM) for the complete list of measures). HBM constructs included perceived susceptibility to [35] and perceived severity of genital HPV infection, cervical cancer, and genital warts, perceived benefits of HPV vaccination [36], and perceived barriers to HPV vaccination [22]. Perceived barriers included vaccine cost and concerns about vaccine safety and side effects. Cost was not combined with safety/ side effects concerns into a single latent factor because items reflected distinct barriers [32]. Whether a doctor or other health care provider had ever recommended the HPV vaccine served as a cue to action.

TPB constructs included attitudes, subjective norms, self-efficacy, and intentions. Attitudes toward HPV vaccination were assessed with the same four items used to assess perceived benefits [35]. Subjective norms reflected the product of normative beliefs for HPV vaccination (i.e., the extent to which their parents, doctor, and best friend think they should be vaccinated) and general motivations to comply with the recommendations of these individuals $[33,37]$. The self-efficacy construct reflected participants' confidence in their ability to receive the vaccine despite potential barriers (e.g., expense, pain) [38]. Intentions to receive the HPV vaccine were assessed with five items that had been validated in previous research [33-34].

HPV vaccine uptake-The primary outcome was self-reported HPV vaccine uptake $(0=$ received no injections; 1 = received $\geq 1$ injection), assessed on the 10-month follow-up 
survey. Participants indicated whether they had received any injections of the HPV vaccine since completing the baseline session (yes or no) and if yes, the number of injections received thus far.

\section{Data Analyses}

We used $t$-tests and chi-square analyses to compare demographics, background variables, and HBM/TPB constructs for participants who completed versus did not complete the follow-up assessment. Chi-square analysis was conducted to assess effects of the intervention (gain, loss, control) on vaccine uptake. Multiple regression analyses were conducted to assess differences in HBM and TPB constructs by experimental condition. In these analyses, the three experimental conditions were represented with two dummy codes (gain vs. control; loss vs. control).

We used structural equation modeling (SEM) to test the HBM versus TPB in predicting HPV vaccine uptake for the full sample. Analyses were conducted with Mplus [39]. Because the primary outcome variable was dichotomous we used a weighted least-squares estimator with mean and variance adjustment (WLSMV). With the exception of physician recommendation and vaccine cost, latent factors were estimated for all model constructs. Factor loadings for all latent variables were significant (all $p s<.001$ ) and coefficient alpha was acceptable for all scales (see Table 1). Thus, the final models for each theory included both a measurement model and a structural model. Models were estimated for each theory with and without intentions as a mediating variable. We also estimated a combined model that predicted vaccine uptake from HBM and TPB constructs simultaneously. Intermediary factors/disturbances were allowed to correlate in all models. For example, in the HBM, perceived susceptibility, perceived severity, perceived benefits, and perceived barriers were allowed to correlate and in the TPB, attitudes, subjective norms, and self-efficacy were allowed to correlate. As we did not have specific hypotheses about which demographic variables would affect perceptions, intentions, and behavior and to provide a cleaner and more parsimonious comparison of the two theories, we did not control for demographics in the models. Model fit was assessed with chi-square, the comparative fit index (CFI), the root-mean-square error of approximation (RMSEA), and the weighted root mean square residual (WRMR). RMSEA values $<.06$ and WRMR values below 1.0 indicate close fit [40]. Formal model comparison tests are not provided, as there is currently no known method for comparing non-nested models estimated with a weighted least-squares estimator; nevertheless, models were evaluated informally by assessing the relative fit and efficacy of each model in predicting uptake. Means, standard deviations, and correlations among model constructs are provided in the ESM.

\section{Results}

\section{Preliminary Analyses}

Attrition analysis-The attrition rate at follow-up was $6 \%(n=43)$. Non-completers were similar to completers on all demographics, background variables, and HBM and TPB constructs with only a few exceptions. Relative to completers, non-completers were more likely to have been recruited from the subject pool, completed fewer years of college, and reported higher perceived susceptibility and subjective norms for HPV vaccination (all $p s<$. $05)$.

Intervention effects-As of the 10-month follow-up, $6 \%(n=41)$ had received one or more injections of the HPV vaccine (one injection: $n=11$; two injections: $n=12$; all three injections: $n=18$ ). Rates of HPV vaccine uptake were equivalent across the three experimental conditions (gain-frame: $n=12$ (5\%); loss-frame: $n=14$ (6\%); control: $n=15$ 
(7\%), $\chi^{2}(2, N=696)=.52, p=.77$. Mean differences in HBM and TPB constructs were observed for perceived susceptibility and perceived benefits/attitudes only. Perceived susceptibility was higher in the loss-framed condition than the control condition, $\beta=.16, t$ $(723)=3.79, p<.001$, and perceived benefits/attitudes were higher in both the gain-framed, $\beta=.15, t(731)=3.49, p<.001$, and loss-framed conditions, $\beta=.11, t(731)=2.56, p=$. 011 , relative to control. To represent the differential effects of the intervention on theory constructs, group differences were estimated (as appropriate) using the same two dummy codes in all of the subsequent SEMs.

\section{Model Comparisons}

Health Belief Model-To test the HBM, we first estimated a model in which perceived susceptibility, perceived severity, perceived benefits, safety concerns, vaccine cost, and physician recommendation were all specified to predict HPV vaccine uptake. The model provided good fit to the data, $\chi^{2}(37)=98.28, p<.001$; CFI=.93; RMSEA=.05, WRMR=. $99 ; \mathrm{R}^{2}$ behavior $=.26$. Significant predictors of uptake included perceived susceptibility ( standardized path coefficient $=.26)$, safety concerns $(-.21)$, vaccine cost $(-.18)$, and physician recommendation (.14, marginal). Perceived severity and perceived benefits were not associated with uptake.

Because the variable representing a cue to action (physician recommendation) reflected advice given to participants before they took part in the intervention and such advice could have influenced their subsequent health beliefs [6], we also estimated an alternative model in which physician recommendation was specified to indirectly affect vaccine uptake via its influence on health beliefs. This model was identical to the previous one except that it also contained paths from physician recommendation to those variables with which it was significantly correlated (perceived susceptibility, perceived benefits, and cost) and the direct path from physician recommendation to uptake was removed (Figure 1). The alternative model provided slightly better fit to the data, $\chi^{2}(39)=82.62, p<.001$; CFI=.95; RMSEA=. 04 , $\mathrm{WRMR}=.84 ; \mathrm{R}^{2}$ behavior $=.26$. Physician recommendation was positively related to perceived susceptibility and perceived benefits and negatively related to cost. As in the previous model, perceived susceptibility, safety concerns, and cost were significant predictors of vaccine uptake.

Next we estimated a model that included intentions as an intermediary predictor of vaccine uptake. This model included paths from physician recommendation to susceptibility, benefits, and cost, paths from the five intermediary variables (susceptibility, severity, benefits, safety concerns, cost) to intentions, and a final path from intentions to uptake. The model provided good fit to the data, $\chi^{2}(47)=115.40, p<.001$; CFI=.90; RMSEA=.05, $\mathrm{WRMR}=.88 ; \mathrm{R}^{2}$ intentions $=.43 ; \mathrm{R}^{2}$ behavior $=.47$. Similar to the model without intentions, physician recommendation was positively related to perceived susceptibility (.12) and perceived benefits (.10) and negatively related to cost (-.12). Additionally, perceived susceptibility (.46), perceived severity (.15), and perceived benefits (.17) were positively related to intentions, whereas safety concerns $(-.19)$ was negatively related. Intentions were positively related to uptake (.68).

Theory of Planned Behavior-To test the TPB, we first estimated a model in which attitudes, subjective norms, and self-efficacy were specified to predict intentions, and intentions and self-efficacy were specified to predict HPV vaccine uptake (Figure 2). The model provided good fit to the data, $\chi^{2}(19)=36.89, p=.008$; CFI=.97; RMSEA=.04, $\mathrm{WRMR}=.75 ; \mathrm{R}^{2}$ intentions $=.57 ; \mathrm{R}_{\text {behavior }}^{2} .50$. Attitudes, subjective norms, and selfefficacy were all positively related to intentions, and intentions were positively related to vaccine uptake. The direct path from self-efficacy to vaccine uptake was not significant. 
Next we estimated a model that excluded intentions as an intermediary predictor of uptake and specified direct paths from attitudes, subjective norms, and self-efficacy to uptake. This model provided good fit to the data, $\chi^{2}(19)=37.38, p=.007$; CFI=.98; RMSEA=.04; $\mathrm{WRMR}=.80 ; \mathrm{R}^{2}$ behavior $=.39$. Subjective norms (.31) and self-efficacy (.46) were positively related to vaccine uptake, but the path from attitudes to uptake was not significant.

Combined model-To test the joint effect of the two theories, we estimated a model in which vaccine uptake was predicted from HBM and TPB constructs simultaneously. Paths were estimated from physician recommendation to those variables with which it was significantly correlated (susceptibility, benefits/attitudes, cost, subjective norms, and selfefficacy). We also estimated paths from susceptibility, severity, benefits, safety concerns, cost, subjective norms, and self-efficacy to uptake (Figure 3 ). The model provided adequate fit to the data, $\chi^{2}(63)=206.37, p<.001 ; \mathrm{CFI}=.87$; RMSEA=.06; $\mathrm{WRMR}=1.05 ; \mathrm{R}^{2}$ behavior $=.43$. Physician recommendation was related to susceptibility, benefits/attitudes, cost, norms, and self-efficacy; however, only subjective norms and self-efficacy emerged as independent predictors of vaccine uptake. It is worth noting that in a model in which we predicted intentions (rather than uptake) from HBM and TPB constructs concurrently, subjective norms (.24) and self-efficacy (.43) remained significant predictors, while three additional predictors emerged: perceived susceptibility (.25), perceived severity (.08), and safety concerns (-.08). Thus, these latter three constructs predicted intentions but not behavior.

\section{Discussion}

Findings from the current study advance health behavior research by testing and comparing two classic theories of health behavior. Although the message framing intervention did not have a direct effect on HPV vaccine uptake, constructs from both the HBM and TPB predicted uptake almost a year later. Significant predictors of vaccine uptake from the HBM included perceived susceptibility and perceived barriers (vaccine safety concerns and vaccine cost). Significant predictors of vaccine uptake from the TPB included intentions, subjective norms, and self-efficacy. Although both theories provided relatively good fit to the data, the TPB consistently outperformed the HBM in predicting vaccination behavior.

Although similarities between the HBM and TPB have been acknowledged [10], the current research is some of the first to rigorously explore those similarities. In the individual models, HBM and TPB constructs accounted for $26 \%$ and $39 \%$ of the variance in vaccine uptake, respectively, suggesting a distinct TPB advantage. However, when constructs were combined into a single model, together HBM and TPB constructs only accounted for $43 \%$ of the variance in uptake. This translates into a $4 \%$ increase in variance explained by HBM constructs over and above that explained by TPB constructs. Although $4 \%$ is by no mean a trivial amount, it does suggest that there is significant overlap in the variance accounted for by constructs associated with these two theories.

Findings from the combined model also highlighted independent predictors of HPV vaccine uptake (subjective norms and self-efficacy), both of which were from the TPB. That subjective norms emerged as an independent predictor of behavior underscores the social nature of health decision-making. When faced with difficult health decisions, individuals look to close others or health care providers for advice. Subjective norms play a particularly important role when the "right" decision is uncertain, as is often the case with preventive health behaviors such as vaccination [24, 26-27]. Results also point to the importance of self-efficacy: Confidence in one's ability to overcome perceived barriers to HPV vaccination was a strong predictor of uptake. As previous studies suggest [12-13], decisions to carry out a health behavior are useful only to the extent that one feels one can adequately implement 
the steps needed to perform the behavior. Although the importance of self-efficacy has been most frequently documented for repeated health behaviors (e.g., physical activity) [12], the current findings confirm that self-efficacy is also important for low frequency health behaviors such as vaccination.

When comparing theories of health behavior it is important to consider the role of proximal versus distal predictors of behavior. One key distinction between the original conceptualizations of the HBM vs. TPB is that the TPB explicitly includes behavioral intentions as a proximal predictor of behavior. By estimating each theory with and without intentions, we were able to observe the sizeable boost in $\mathrm{R}^{2}$ behavior when intentions were included in the model. Indeed, consistent with the TPB, intentions emerged as the strongest predictor of vaccine uptake, accounting for approximately $50 \%$ of the variance in behavior. Results suggest that by including intentions - a very proximate factor underlying behavior one is bound to increase the efficacy of the model. Hence, when comparing theories of health behavior, it seems appropriate to level the playing field by either including or excluding intentions from each theory being compared.

The current study raises the interesting possibility that cues to action might operate via their effects on health-related perceptions (e.g., beliefs, attitudes). The few studies investigating cues to action have reported mixed results with some suggesting that cues to action have direct effects on health behavior and others indicating null effects $[6,13]$. The equivocal findings may be due to different operationalizations of the construct across studies (e.g., physician advice, exposure to mass media campaigns, reminder postcards, knowing someone with a particular medical condition). Another possibility is that, in addition to having direct effects on behavior, cues to action may influence behavior indirectly via their effects on health-related perceptions [6]. Indeed, participants who were advised by a health care provider to receive the HPV vaccine reported higher perceived susceptibility to HPV infection, greater perceived benefits and lower perceived barriers to HPV vaccination, higher self-efficacy, and more favorable subjective norms for receiving the vaccine.

Moreover, the two independent predictors of uptake from the combined model (norms, selfefficacy) were predicted by physician recommendation. Both predictors are from the TPB, suggesting that cues to action may be represented informally in the TPB via these constructs. It will be important for future studies to investigate the possibility that cues to action affect behavior indirectly via their effects on health-related perceptions.

Although the longitudinal design was a strength of the current research, the data are ultimately correlational. Weinstein outlined potential problems associated with testing health behavior theories with correlational data [30]. He noted that inflated relationships between perceptions and behavior are most likely to emerge for ongoing or repeated health behaviors (e.g., physical activity, condom use) because, although perceptions may cause future behavior, they are also likely to reflect previous experience with the behavior. Because the present study was limited to women who had not initiated the series, the "behavior causing perceptions" problem is somewhat lessened. Nevertheless, because baseline data were collected over two years after the HPV vaccine was widely available, women's attitudes and beliefs may have reflected their lack of action (i.e., the fact that they had not received the vaccine). Stronger causal inferences would be obtained by examining newly available health behaviors or by manipulating health behavior theory constructs experimentally $[17,30]$.

Findings have important implications for future HPV vaccination interventions with young adult women. Results point to potentially modifiable perceptions that may be promising candidates to target in future interventions (e.g., perceived susceptibility, perceived barriers such as vaccine safety concerns and cost, subjective norms, self-efficacy). As self-efficacy and perceived barriers are closely linked, it will be important to understand key barriers to 
HPV vaccination. Previous research [33] suggests that women who are not sexually active and women who are in a monogamous relationship report relatively low perceived susceptibility to HPV infection. Because risk perceptions are intimately tied to both relationship status and sexual history, increasing such perceptions will likely be a challenge for future interventions. Finally, physician recommendation was a significant predictor of HPV-related perceptions (e.g., perceived susceptibility, subjective norms). Given the importance of physician advice for vaccination behavior [28], future interventions should address this essential component as well.

Limitations of the present study provide useful directions for future research. One limitation pertains to our measure of attitudes. In the TPB, attitudes are defined as the product of (a) one's beliefs about outcomes associated with engaging in the health behavior and (b) one's evaluations of those outcomes (i.e., whether they are viewed as positive or negative). Our measure focused more on outcome beliefs than on outcome evaluations and thus may not have fully captured the value participants place on avoiding outcomes associated with HPV infection. A second limitation involves our use of self-efficacy as a marker for perceived behavioral control. Although self-efficacy is highly correlated with perceived behavioral control [e.g., 1, 9-10], perceived behavioral control is a broader concept that reflects perceptions about the extent to which environmental factors affect the ease or difficulty of performing the behavior. Thus our measure of self-efficacy captured only one aspect of perceived behavioral control. Third, findings are limited to a single low frequency health behavior within a single convenience sample. Different results may have been observed for a preventive health behavior that requires regular and repeated action (e.g., physical activity). Additional research is needed to assess the generalizability of these findings, both in terms of other health behaviors as well as other populations (e.g., girls younger than age 18). Fourth, because we examined health behavior theories that focus on individual level predictors, findings do not address health care system or policy level factors that may affect HPV vaccination. Fifth, vaccine uptake was relatively low (6\%). Although previous studies have documented somewhat higher rates of uptake, we do not think the current result is anomalous, because the length of our follow-up period was relatively short. Nevertheless, the low rate of uptake could have implications for over-fitting our statistical models. Finally, HPV vaccine uptake was self-reported rather than verified by participants' medical records.

Theories of health behavior provide conceptual frameworks for understanding the determinants of health behavior and developing behavioral interventions. The current study compared two classic theories of health behavior in their ability to predict HPV vaccination. Findings indicated a clear advantage of the TPB over the HBM, although the two theories shared a great deal of overlap. Results suggest promising avenues for theory testing in future research.

\section{Supplementary Material}

Refer to Web version on PubMed Central for supplementary material.

\section{Acknowledgments}

We thank members of the Women's Health research team (Khadija Andrews, Kristina Banda, Kathleen Burns, Michelle DiMarco, Sophia Harvey, Elizabeth Howe, Jessica Kraich, Lisa Langenderfer, Meghan McLeod, Mallory McRoberts, Kristina Martinez, Shella Mesa, Samantha O'Hara, Virginia Parker, Victoria Patronis, Michelle Poole, Alana Resmini, and Jennifer Rice, Stephanie Urena, \& Becky Wiesenfeld) for their assistance with this project. This research was supported in part by a grant from the National Cancer Institute of the National Institutes of Health (R03-CA138069). 


\section{References}

1. Ajzen I. The theory of planned behavior. Organ Behav Hum Decis Process. 1991; 50:179-211.

2. Becker MH, Maiman LA. Sociobehavioral determinants of compliance with health and medical care recommendations. Med Care. 1975; 13:10-24. [PubMed: 1089182]

3. Conner, M.; Norman, P., editors. Predicting health behavior. 2. Buckinghman, UK: Open University Press; 2005.

4. Fishbein, M.; Ajzen, I. Belief, attitude, intention, and behavior: An intro to theory and research. Reading, MA: Addison-Wesley; 1975.

5. Rosenstock IM, Strecher VJ, Becker MH. Social learning theory and the health belief model. Health Educ Q. 1988; 15:175-183. [PubMed: 3378902]

6. Abraham, C.; Sheeran, P. Health belief model. In: Conner, M.; Norman, P., editors. Predicting health behavior. Buckinghman, UK: Open University Press; 2005. p. 28-80.

7. Conner, M.; Sparks, P. Theory of planed behaviour and health behavior. In: Conner, M.; Norman, P., editors. Predicting health behavior. Buckinghman, UK: Open University Press; 2005. p. 171-222.

8. Rosenstock IM. Historical origins of the health belief model. Health Educ Monogr. 1974; 2:328335.

9. Noar SM, Zimmerman RS. Health Behavior Theory and cumulative knowledge regarding health behaviors: Are we moving in the right direction? Health Educ Res. 2005; 20:275-290. [PubMed: 15632099]

10. Weinstein ND. Testing four competing theories of health-protective behavior. Health Psychol. 1993; 12:324-333. [PubMed: 8404807]

11. Albarracin D, Johnson BT, Fishbein M, Muellerleile PA. Theories of reasoned action and planned behavior as models of condom use: A meta-analysis. Psychol Bull. 2001; 127:142-161. [PubMed: 11271752]

12. Brewer, NT.; Rimer, BK. Perspectives on health behavior theories that focus on individuals. In: Glanz, K.; Rimer, BK.; Viswanath, K., editors. Health behavior and health education: Theory, research, and practice. San Francisco, CA: Jossey-Bass; 2008. p. 149-165.

13. Champion, VL.; Skinner, CS. The health belief model. In: Glanz, K.; Rimer, BK.; Viswanath, K., editors. Health behavior and health education: Theory, research, and practice. San Francisco, CA: Jossey-Bass; 2008. p. 45-65.

14. Bonetti D, Johnston M, Clarkson J, Turner S. Applying multiple models to predict clinicians' behavioural intention and objective behaviour when managing children's teeth. Psychol Health. 2009; 24:843-860. [PubMed: 20205030]

15. McClenahan C, Shevlin M, Adamson G, Bennett C, O'Neill B. Testicular self-examination: a test of the health belief model and the theory of planned behaviour. Health Educ Res. 2007; 22:272284. [PubMed: 16885203]

16. Tavares LS, Plotnikoff RC, Loucaides C. Social-cognitive theories for predicting physical activity behaviours of employed women with and without young children. Psychol Health Med. 2009; 14:129-142. [PubMed: 19235072]

17. Weinstein ND, Rothman AJ. Commentary: Revitalizing research on health behavior theories. Health Educ Res. 2005; 20:294-297. [PubMed: 15632097]

18. Markowitz LE, Dunne EF, Saraiya M, et al. Quadrivalent Human Papillomavirus Vaccine: Recommendations of the Advisory Committee on Immunization Practices (ACIP). MMWR Morb Mortal Wkly Rep. 2007; 56:1-24. [PubMed: 17218934]

19. Centers for Disease Control and Prevention. FDA licensure of bivalent human papillomavirus vaccine (HPV2, Cervarix) for use in females and updated HPV vaccination recommendations from the Advisory Committee on Immunization Practices (ACIP). MMWR Morb Mortal Wkly Rep. 2010; 59:626-629. [PubMed: 20508593]

20. Future II Study Group. Quadrivalent vaccine against human papillomavirus to prevent high-grade cervical lesions. N Engl J Med. 2007; 356:1915-1927. [PubMed: 17494925]

21. Paavonen J, Naud P, Salmeron J, et al. Efficacy of human papillomavirus (HPV)-16/18 AS04adjuvanted vaccine against cervical infection and precancer caused by oncogenic HPV types 
(PATRICIA): Final analysis of a double-blind, randomised study in young women. Lancet. 2009; 374:301-314. [PubMed: 19586656]

22. Brewer NT, Fazekas KI. Predictors of HPV vaccine acceptability: A theory-informed, systematic review. Prev Med. 2007; 45:107-114. [PubMed: 17628649]

23. Zimet GD, Liddon N, Rosenthal SL, Lazcano-Ponce E, Allen B. Chapter 24: Psychosocial aspects of vaccine acceptability. Vaccine. 2006; 24(Suppl 3):S3/201-209. [PubMed: 16950008]

24. Allen JD, Mohllajee AP, Shelton RC, et al. Stage of adoption of the human papillomavirus vaccine among college women. Prev Med. 2009; 48:420-425. [PubMed: 19133288]

25. Brewer NT, Gottlieb SL, Reiter PL, et al. Longitudinal predictors of HPV vaccine initiation among adolescent girls in a high-risk geographic area. Sex Transm Dis. 2011; 38:197-204. [PubMed: 20838362]

26. Conroy K, Rosenthal SL, Zimet GD, et al. HPV vaccine uptake, predictors of vaccination, and selfreported barriers to vaccination. J Womens Health. 2009; 18:1679-1686.

27. Roberts ME, Gerrard M, Reimer R, Gibbons FX. Mother-daughter communication and human papillomavirus vaccine uptake by college students. Pediatrics. 2010; 125:982-989. [PubMed: 20385645]

28. Rosenthal SL, Weiss TW, Zimet GD, et al. Predictors of HPV vaccine uptake among women aged 19-26: Importance of a physician's recommendation. Vaccine. 2011; 29:890-895. [PubMed: 20056186]

29. Reiter PL, Brewer NT, Gottlieb SL, McRee AL, Smith JS. Parents' health beliefs and HPV vaccination of their adolescent daughters. Soc Sci Med. 2009; 69:475-480. [PubMed: 19540642]

30. Weinstein ND. Misleading tests of health behavior theories. Ann Behav Med. 2007; 33:1-10. [PubMed: 17291165]

31. Gerend MA, Shepherd JE. Correlates of HPV knowledge in the era of HPV vaccination: A study of unvaccinated young adult women. Women Health. 2011; 51:25-40. [PubMed: 21391159]

32. Gerend MA, Shepherd MA, Shepherd JE. The multidimensional nature of perceived barriers: global versus practical barriers to HPV vaccination. Health Psychol. in press.

33. Gerend MA, Shepherd JE. Using message framing to promote acceptance of the human papillomavirus vaccine. Health Psychol. 2007; 26:745-752. [PubMed: 18020847]

34. Gerend MA, Shepherd JE, Monday KA. Behavioral frequency moderates the effects of message framing on HPV vaccine acceptability. Ann Behav Med. 2008; 35:221-229. [PubMed: 18347893]

35. Weinstein ND, Kwitel A, McCaul KD, et al. Risk perceptions: assessment and relationship to influenza vaccination. Health Psychol. 2007; 26:146-151. [PubMed: 17385965]

36. Fazekas KI, Brewer NT, Smith JS. HPV vaccine acceptability in a rural Southern area. J Womens Health. 2008; 17:539-548.

37. Boehner CW, Howe SR, Bernstein DI, Rosenthal SL. Viral sexually transmitted disease vaccine acceptability among college students. Sex Transm Dis. 2003; 30:774-778. [PubMed: 14520177]

38. Schwarzer R, Renner B. Social-cognitive predictors of health behavior: Action self-efficacy and coping self-efficacy. Health Psychol. 2000; 19:487-495. [PubMed: 11007157]

39. Muthén, B.; Muthén, L. Mplus Version 3.12. 1998.

40. Newsom, J. [Accesibility verified March 12, 2011.] Practical approaches to dealing with nonnormal and categorical variables. Available at http://www.upa.pdx.edu/IOA/newsom/semclass/ho_estimate2.pdf 


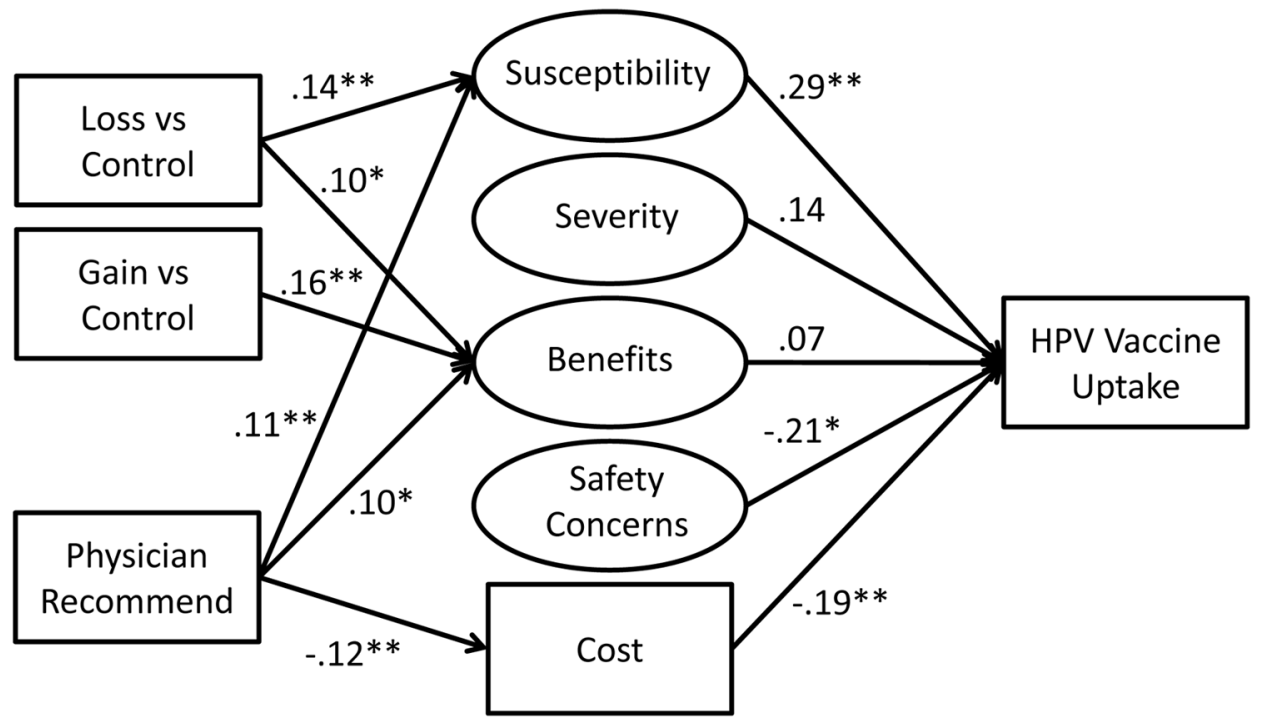

Figure 1.

Prediction of HPV vaccine uptake from Health Belief Model (HBM) constructs. HBM constructs were assessed immediately after exposure to the message framing intervention. HPV vaccine uptake was assessed approximately 10 months later.

$* p<.05 * * p<.01$ 


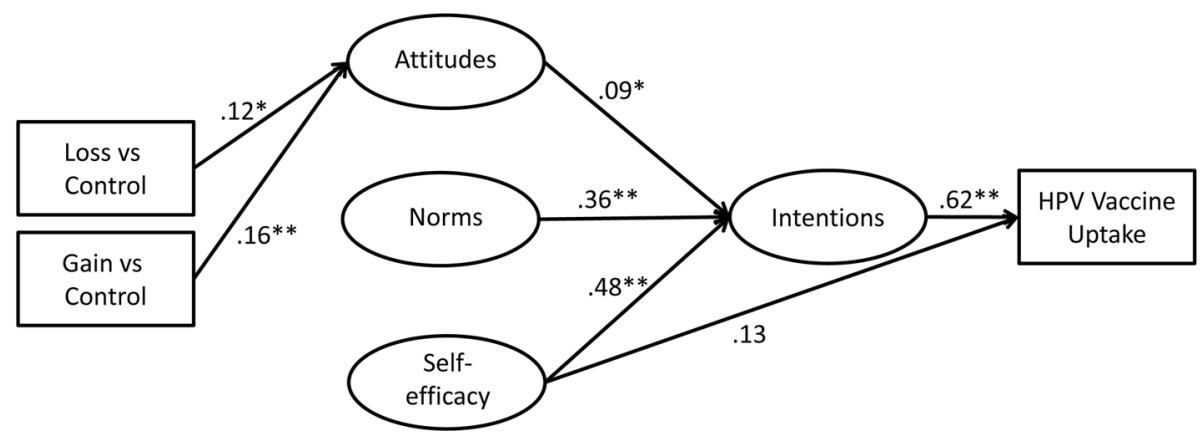

Figure 2.

Prediction of HPV vaccine uptake from Theory of Planned Behavior (TPB) constructs. TPB constructs were assessed immediately after exposure to the message framing intervention. HPV vaccine uptake was assessed approximately 10 months later.

$* p<.05 * * p<.01$ 


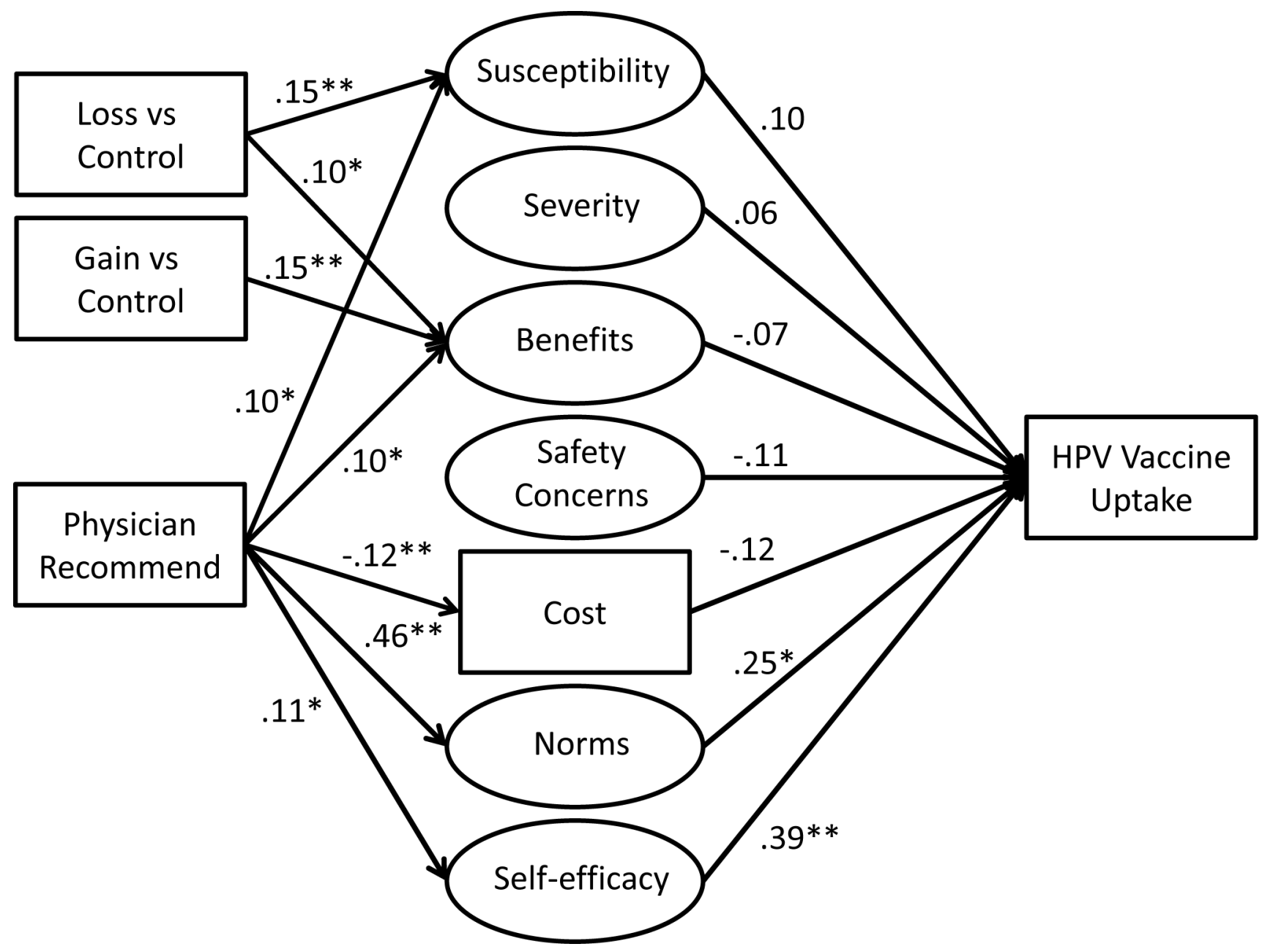

Figure 3.

Prediction of HPV vaccine uptake from Health Belief Model (HBM) and Theory of Planned Behavior (TPB) constructs combined. HBM and TPB constructs were assessed immediately after exposure to the message framing intervention. HPV vaccine uptake was assessed approximately 10 months later.

$* p<.05 * * p<.01$ 
Table 1

Example Items Used to Assess Health Belief Model (HBM) and Theory of Planned Behavior (TPB) Constructs

\begin{tabular}{|c|c|c|c|}
\hline Construct & \# items & $a$ & Example item (response options) \\
\hline \multicolumn{4}{|l|}{ Health Belief Model } \\
\hline Perceived susceptibility & $9^{a}$ & .94 & $\begin{array}{l}\text { If you don't get vaccinated for HPV, how likely is it that you'll [become infected with } \\
\text { genital HPV; get cervical cancer; get genital warts] in the future? (1=very unlikely-7=very } \\
\text { likely) }\end{array}$ \\
\hline Perceived severity & $9^{a}$ & .91 & $\begin{array}{l}\text { [Being infected with HPV; Having cervical cancer; Having genital warts] would have } \\
\text { major consequences on my life. (1=disagree strongly-7=agree strongly) }\end{array}$ \\
\hline Perceived benefits & 4 & .88 & $\begin{array}{l}\text { Getting vaccinated for HPV will help protect me from genital HPV infection. (1=disagree } \\
\text { strongly-7=agree strongly) }\end{array}$ \\
\hline \multicolumn{4}{|l|}{ Perceived barriers } \\
\hline Cost & 1 & -- & $\begin{array}{l}\text { How much would the following factors prevent you from getting vaccinated for HPV? If } \\
\text { the vaccine cost too much. (1=disagree strongly-7=agree strongly) }\end{array}$ \\
\hline Safety concerns & 2 & .87 & $\begin{array}{l}\text { How much would the following factors prevent you from getting vaccinated for HPV? } \\
\text { Concerns about possible side effects of the vaccine. (1=disagree strongly- } 7=\text { agree strongly) }\end{array}$ \\
\hline \multicolumn{4}{|l|}{ Cue to action } \\
\hline Physician recommendation & 1 & -- & $\begin{array}{l}\text { Has a doctor or other health care provider ever recommended that you get the HPV } \\
\text { vaccine? (yes; no) }\end{array}$ \\
\hline \multicolumn{4}{|l|}{ Theory of Planned Behavior } \\
\hline Attitudes & 4 & .88 & $\begin{array}{l}\text { Getting vaccinated for HPV will help protect me from genital HPV infection. (1=disagree } \\
\text { strongly-7=agree strongly) }\end{array}$ \\
\hline Subjective norms & 6 & .78 & $\begin{array}{l}\text { How much [do your parents; does your doctor; does your best female friend] think you } \\
\text { should receive the HPV vaccine? }(-2=\text { not at all; }-1=\mathrm{a} \text { little; } 0=\text { somewhat; }+1=\mathrm{a} \text { lot; }+2=\mathrm{a} \\
\text { great deal) } \\
\text { In general, I want to do what my [parents think; doctor thinks; best female friend thinks] I } \\
\text { should do. ( } 0=\text { not at all; } 1=\text { a little; } 2=\text { somewhat; } 3=\mathrm{a} \text { lot; } 4=\text { a great deal) }\end{array}$ \\
\hline Self-efficacy & 3 & .85 & $\begin{array}{l}\text { I feel confident in my ability to get vaccinated for HPV, even if it is expensive. ( } 1=\text { disagree } \\
\text { strongly-7=agree strongly) }\end{array}$ \\
\hline Intentions & 5 & .96 & $\begin{array}{l}\text { In the next year, how likely is it that you'll try to get more information about the HPV } \\
\text { vaccine? (1=very unlikely-7=very likely) }\end{array}$ \\
\hline
\end{tabular}

a Perceived susceptibility and perceived severity to three different HPV-related outcomes (genital HPV infection; cervical cancer; genital warts) were assessed with three different questions for each construct. Three 3-item composites were computed for each HPV-related outcome for each construct. These three composites for perceived susceptibility and perceived severity were then used as indicators for the latent factors of perceived susceptibility and perceived severity, respectively, in the models. $a=$ Coefficient alpha. 